\title{
Brain-inspired spiking neural network controller for a neurorobotic whisker system
}

\author{
Alberto Antonietti ${ }^{1,2 凶}$, Alice Geminiani $^{1}$, Edoardo Negri $^{1,2}$, Egidio D’Angelo $^{1,3}$, Claudia Casellato $^{1^{*}}$, and Alessandra \\ Pedrocchi $^{2^{*}}$ \\ ${ }^{1}$ Neurocomputational laboratory, Department of Brain and Behavioral Sciences, University of Pavia, Pavia, Italy \\ ${ }^{2}$ Nearlab, Department of Electronics, Information and Bioengineering, Politecnico di Milano, Milano, Italy \\ ${ }^{3}$ Brain Connectivity Center, IRCCS Mondino Foundation, Pavia, Italy \\ *Co-last Authors
}

\begin{abstract}
It is common for animals to use self-generated movements to actively sense the surrounding environment. For instance, rodents rhythmically move their whiskers to explore the space close to their body. The mouse whisker system has become a standard model to study active sensing and sensorimotor integration through feedback loops. In this work, we developed a bioinspired spiking neural network model of the sensorimotor peripheral whisker system, modelling trigeminal ganglion, trigeminal nuclei, facial nuclei, and central pattern generator neuronal populations. This network was embedded in a virtual mouse robot, exploiting the Neurorobotics Platform, a simulation platform offering a virtual environment to develop and test robots driven by brain-inspired controllers. Eventually, the peripheral whisker system was properly connected to an adaptive cerebellar network controller. The whole system was able to drive active whisking with learning capability, matching neural correlates of behaviour experimentally recorded in mice.
\end{abstract}

Point neuron model | neurorobotic architecture | active whisking | Vibrissae | Trigeminal Ganglion | Trigeminal Nuclei | Facial Nuclei | CPG

Correspondence: alberto.antonietti@polimi.it

\section{Introduction}

A fundamental question in system neuroscience is to identify how peripheral sensory stimuli are processed in multiple brain regions showing specific neuronal activity. Rodent whisker-mediated touch system is a structurally well-known system which gives rise to complex adaptive behaviours (1). Specifically, the rodent whisker system represents an efficient combination of active perception and sensorimotor integration, in which self-generated movements are used to actively sense their environment, i.e., scanning the surrounding to collect behaviourally-relevant information. Rodents have specialized muscles in their mystacial pad able to control the hair position (2). They rhythmically protract their whiskers swiping the space surrounding the head and gathering information about shape and position of objects around them.

In the rodent whisker system, the primary afferences come from the trigeminal ganglion (TG) and the efferences project to motoneurons in the facial nuclei (FN). There are no direct connections between the two, indeed the innermost feedback loop is a di-synaptic reflex at brainstem level, involving interneurons from the trigeminal nuclear complex (TN) $(3,4)$. Outer loops in the whisker system involve multiple brain regions: the cerebellum, the midbrain (superior colliculus), and forebrain (5). The most extensive and most studied cortical loop is the whisker-barrel loop, involving the vibrissa primary sensory and motor cortex (6). Therefore, this somatosensory system is an ideal candidate to investigate the link between circuitry and function and to understand the underlying neuronal mechanisms in sensory readout and information processing.

Computational models of this system can play a fundamental role for multi-scale investigations, from neuron to behaviour, also thanks to availability of multi-scale experimental data in rodents for constraining and validating the models. In this work, we have developed a Spiking Neural Network (SNN) model able to process information encoded during whisking using a time coding representation of neuronal activity (710). While other models and kinds of artificial neural networks (e.g., rate-based or mean field models) are very powerful tools, based on brain dynamics, we choose SNNs because they are closer to biological reality, since they mimic the way information is coded and transmitted inside a real brain. In this work, each neuron in the network have been modeled with the most simplified spiking model, which is the Integrate \& Fire model (I\&F). SNNs can learn patterns of activity thanks to embedded plasticity models: here we included a Spike-Timing Dependent Plasticity (STDP) model (11-15) in the cerebellar circuit, which was inserted in the control system (outer loop) to test learning capabilities.

Neurorobotic models of rodent whisking. Models of brain regions embedded in neurorobots allow to reproduce the functional mechanisms of living beings in closed perception-action loops $(16,17)$. Various examples of neurorobots using biologically inspired whiskers have been implemented in the last years. Among them, it is worth to cite the Whiskerbot, the SCRATCHbot and the Shrewbot (18).

The Whiskerbot consists of a robotic platform constituted by a head sensory unit of 150x170 mm and a two-wheeled body. The head carries six whiskers per side arranged in rows of three. Analogue information from whisker deflection is converted in empirically-based spike trains. It is able to freely move in an environment, actively whisking and orient toward salient stimuli using a neural network model of the superior colliculus $(18,19)$. The SCRATCHbot has a larger number of whiskers and degrees of freedom to position them in the environment. It was developed to reproduce different models of whisking pattern generation and to actively explore its environment using a simple model of tactile atten- 
tion $(18,20)$. Both these robots were further enriched integrating the Shrewbot platform, which introduced algorithms able to detect texture and object from an active whisker array $(18,21,22)$.

Real neurorobots are excellent test benches to challenge a neuro-inspired controller to demonstrate its capabilities, especially because of the real time computation constraints and the noise of the physical hardware and equipment, both intrinsic (non-ideal electronics sensors, limited spatio-temporal resolution, delays) and extrinsic (unexpected changes in the environment, external perturbing forces/torques, etc.). However, the implementation of physical neurorobots is complex and expensive, therefore limiting their adoption by neuroscientists to test computational models of the brain circuitry. Besides, it is also difficult to replicate the obtained results without an exact replica of the equipment used. Finally, the brain-inspired circuit controlling the robot can have a limited complexity, in terms of realism (neuronal models), number of elements (neurons and synapses), activity (spike events), and functionality (e.g., short and long term plasticity rules) for the sake of limited computational load required for real-time computations.

In this paper, we have developed a biologically-inspired neurorobotic whisker system on a virtual mouse inside the Neurorobotics Platform (NRP) (23-26). This work focuses on the reproduction of the peripheral parts of the whisker sensorimotor system and on the integration of the sensory inputs with an adaptive cerebellar spiking controller to perform a spatial learning task.

\section{Materials and methods}

In this section, anatomy and physiology of the rodent whisker system are described and for each peripheral component (active vibrissae, sensory pathway, motor pathway, and trigeminal loop) the neurorobotic implementation is reported. Then the protocol to test the whisking controller, including an adaptive cerebellar network, is described, tailored on the experimental paradigms used on mice to understand neural mechanisms of active whisking and reward-based learning. Finally, the software libraries and computing resources are reported.

\section{Rodent whisker system and its neurorobotic imple-} mentation. Given the low number of degrees of freedom involved and the ease to make tests in laboratory conditions, the rodents whisker system has become a popular model for studying brain development, experience-dependent plasticity, active sensation, motor control, and sensorimotor integration $(2,5)$.

We have implemented the physical and the neural elements that constitute the whisker system of a rodent. The first step was the implementation of active whiskers (or vibrissae) in the mouse robot, making them controllable and allowing the reading of dynamic and kinematic parameters and information about the contact with external objects. Then, it was necessary to read inputs from the simulated environment and encode them realistically in the behaviour of vibrissal affer- ents. Once unprocessed data were gathered from afferents, further elaboration steps were carried out. These processed signals were used to directly control the motor actions, thus closing the first sensorimotor feedback loop, or extracting higher level information such as the phase of the whisking when a contact happened.

Active vibrissae. Vibrissae are long and sensitive hairs common to most mammals, including all primates except humans (27). Mystacial vibrissae are the ones growing on the mystacial pad, located at the sides of the animal snout, and have a major role in tactile spacial sensing and object discrimination (28).

\section{Neurorobotic implementation}

To implement sensible whiskers in the mouse robot model inside the Neurorobotic Platform, we started from the HBP Mouse Robot v2 (dimensions: $140 \mathrm{~cm}$ from the nose tip to the end of the tail, $35 \mathrm{~cm}$ width, $35 \mathrm{~cm}$ height). The 3D models of the whiskers were defined as rigid cylinders: two right and two left whiskers, anchored to the mouse nose and with two different lengths and roll angles (Figure 1A). The lower whiskers (L0 and R0 for left and right whisker, respectively) are longer $\left(50 \mathrm{~cm}\right.$ each) and are rotated of $11^{\circ}$, while the upper whiskers (L1 and R1) are shorter ( $25 \mathrm{~cm}$ each) and are rotated of $22^{\circ}$. All whiskers have a diameter of $1 \mathrm{~cm}$ and their position can be independently controlled setting a torque at the revolving joints that link them to the mouse nose.

Sensory pathway. In the rodent whisker system (Figure 1B), the primary afferents have their nucleus located in the trigeminal ganglion.

When an object enters the peri-personal space around the head of a rodent, it will be sensed by the moving whiskers and its position and shape are encoded by neuronal signals. This can be done directly by the primary afferents or after some processing inside inner brain structures. The way the position of an external object is represented inside the vibrissal system of rodents is called vibrissal location coding (29).

The spatial organization of whiskers in the mystacial pad varies between different mammals, but are quite similar between rats and mice (28). Rats and mice whiskers are aligned in 5 rows, the upper 2 have four whiskers each, while the lower 3 counts 7 whiskers each (5). From rostral to caudal in each vibrissal row, the whisker length increases exponentially (28).

In the vibrissal follicle, three types of mechanoreceptors are present: Merkel cells, lanceolate endings and free nerve endings (5). Merkel cells ending are slowing adapting mechanoreceptors and they mostly signal ongoing movements, while lanceolate endings are rapidly adapting and respond to fast changes.

Mechanoreceptors surrounding each whisker transmit sensory information through cells whose bodies are located in the TG. Each neuron sends signals from a single vibrissa, while each follicle is innervated by $\sim 200$ TG neurons.

A main distinction between trigeminal ganglion cells can be drawn based on the kind of receptor they receive information from: rapidly adapting or slowly adapting. While measuring 

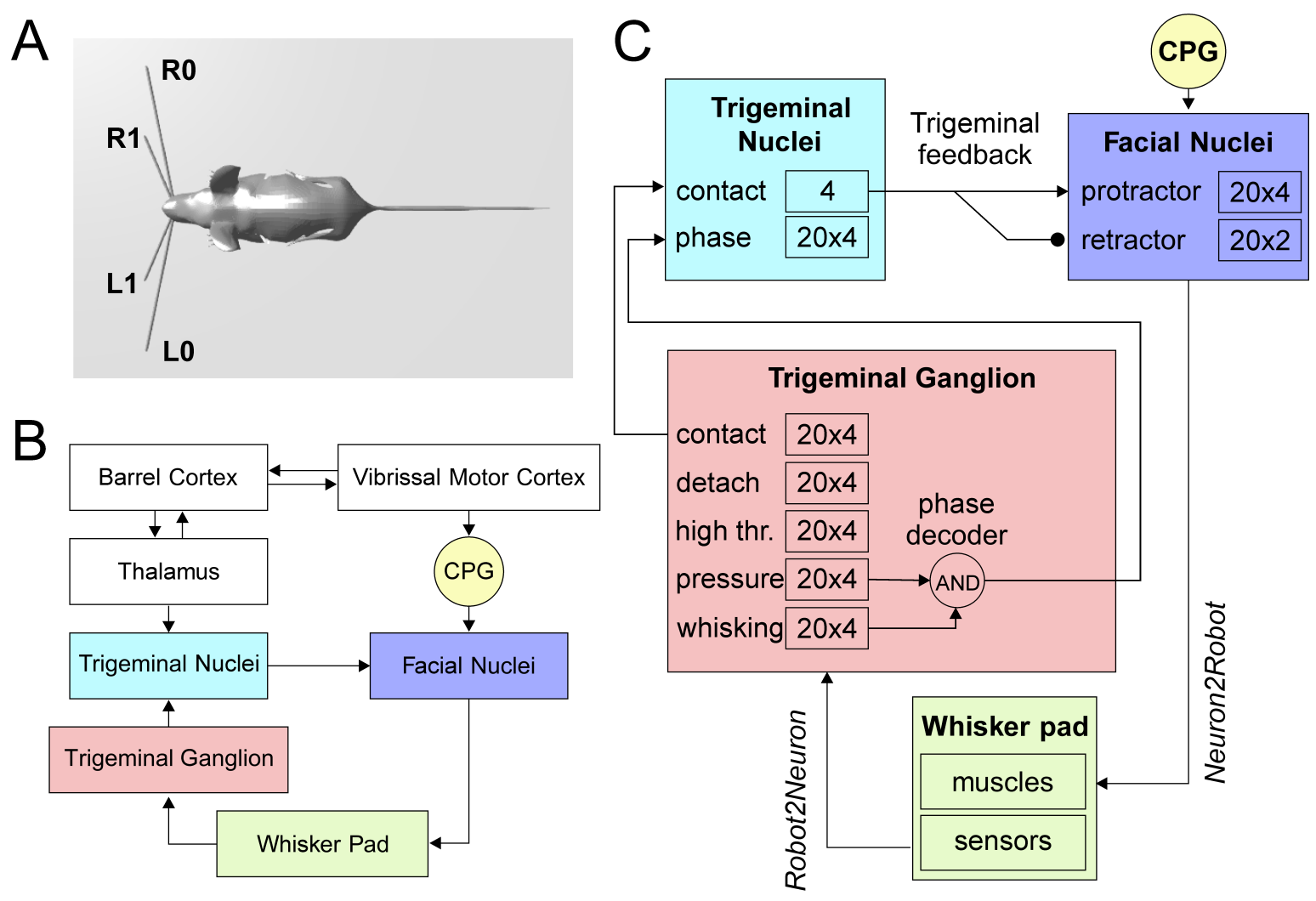

Fig. 1. The rodent whisker system. A) Virtual robotic mouse implemented in the NRP, with two whiskers per side. L0 and R0 are the lower left and right whiskers, L1 and R1 are the upper whiskers. B) Block diagram of the rodent whisker system, including sensory and motor pathways, and its integration with higher-order areas (thalamus and cortex). C) SNN implementation of the mouse peripheral whisker system; numbers in each block represent the size of the neural populations included in that brain region. Arrows represent excitatory connections, circles inhibitory connections.

cell activities in-vivo during free whisking, it can be observed that slowly adapting cells fire more during whisking in air. In fact, when contacting an object, all cells increase their firing rate, but the increase is more consistent for rapidly adapting cells (30).

In 2003, Szwed et al. induced artificial whisking in rats and measured the activity of TG cells (31). According to their results, TG neurons can be classified into distinct categories based on their responses to whisking in air and against an object:

- Touch cells responding only when the whisker touches an object, they can be further divided into contact cells, responding only at the beginning of the contact, detach cells, only at the end of the contact, and pressure cells with a tonic force-dependent response.

- Whisking cells responding only on whisker movement and not on object contact (if the contact does not affect the movement of the follicle).

- High threshold cells responding only to strong mechanical stimulations.

\section{Neurorobotic implementation}

The Neurorobotics Platform permits to connect the environment to the robot sensors and actuators using so called transfer functions. These are Python functions defining to and from which ROS topics and neural populations read and write. They can be defined as Robot 2 Neuron (sensory) or Neuron2Robot (motor) according to the direction of the information flow.

The transfer function written to implement the follicle sensors is of the Robot 2 Neuron kind, since it reads the information on whisker mechanical status and position, and then process the data to obtain: possible contacts of a whisker against an object, the contact distance from the snout, the whisker angular position. The input transfer function is connected to the trigeminal ganglion neurons (Figure 1C), divided in five populations (TG pressure, TG high threshold, TG contact, TG detach, and TG whisking cells) with 20 neurons per whisker each, based on the functional separation observed in literature (31).

TG high threshold cells fire with a fixed rate when the contact is very close to the snout (less then $2 \mathrm{~cm}$ ), constituting $d e$ facto a labelled line encoding for proximity, and TG whisking cells encode the current whisker position, each neuron has a Gaussian-shaped sensitivity and fire when the whisker position is within a narrow range around its maximum sensitive angle.

Motor pathway. The head of a rodent exploring its peripersonal space is constantly moving, side-to-side and up-anddown, while its nose moves side-to-side and the whiskers scan back-and-forth. These movements have a rhythmic component phase locked to sniffing, centered around $7 \mathrm{~Hz}$ in rats and $11 \mathrm{~Hz}$ in mice (6). 
The representation of vibrissae in the primary motor cortex occupies around $20 \%$ of motor cortical area. There is no accepted topographic map, some studies obtained single-whisker responses, others observed how the number of whiskers showing evoked movements changes with the level of anesthesia used. In-vivo single cell microstimulation consistently evoked multi-whisker movements. There is strong evidence that the primary motor cortex controls only indirectly the muscle activity projecting to brainstem premotor networks, acting as central pattern generators (CPG) (32).

Motor neurons controlling muscles of the whisker pad are located in the lateral FN and send motor commands via the facial nerve. About $80 \%$ of the FN neurons evoking whisker movements induce protractions of a single whisker and about $20 \%$ the retraction of multiple whiskers (5).

\section{Neurorobotic implementation}

The CPG has been implemented in the robot mouse as a single neuron. Controlled by a Robot 2 Neuron transfer function, the CPG neuron emits regular spikes at a constant frequency in lower-theta band $(4 \mathrm{~Hz})$. It is connected with excitatory synapses to both protractors and retractors neurons, with delays of $1 \mathrm{~ms}$ and $50 \mathrm{~ms}$ respectively, in order to generate a rhythmic whisking movement .

Facial nuclei (Figure 1C) are divided into protractors and retractors. Protractors have been implemented with four populations of 20 neurons, where each population controls one whisker (L0, L1, R0, and R1). For retractors there are just two populations, one for each side (one population for L0 and L1, and one population R0 and R1). Sizes of populations are based on biological evidences, as described before.

The spiking activity of protractors and retractors is then transformed into a torque signal, applied to each whisker, using (1).

$$
\operatorname{torque}(t)=\alpha_{\text {pro }} \cdot F R_{\text {pro }}(t)-\alpha_{\text {ret }} \cdot F R_{\text {ret }}(t)
$$

Where $F R_{\text {pro }}(t)$ and $F R_{\text {ret }}(t)$ are the instantaneous firing rates of protractors and retractors, respectively (in $\mathrm{Hz}$ ), while $\alpha_{\text {pro }}$ and $\alpha_{\text {ret }}$ are constant gains, set to $0.15 \mathrm{Nm} / \mathrm{Hz}$ and 0.10 $\mathrm{Nm} / \mathrm{Hz}$, respectively.

Trigeminal loop. The trigeminal loop in the brainstem is a second order loop and is the most peripheral of the various loops constituting the vibrissal sensorimotor system. On the afferent side, neurons in the TG gather information from the follicles and project with excitatory synapses to the trigeminal nuclei complex. On the efferent side, subcortical whisking centers and CPG send motor commands to the motoneurons in the FN (3). Facial motoneurons driving muscles to protract the vibrissae receive a short latency input $(7.5 \pm 0.4$ $\mathrm{ms}$ ) followed by synaptic excitation from neurons in TN. These connections result in a pull-push mechanism allowing for rapid modulation of vibrissa touch during exploration.

\section{Neurorobotic implementation}

When a whisker touches an object, the physical simulator makes it bounce according to physical properties of the simulated materials, producing a noisy contact signal. This offers us the possibility to apply the trigeminal feedback mech- anisms previously described as a biologically inspired debouncing mechanism. First, a neural population (TN contact) has been created in the trigeminal nuclei, of the same size of TG contact and taking from it excitatory, one-to-one connections (Figure 1C). Then, TN contact neurons were connected to the facial nuclei protractors with excitatory synapses having a $7.5 \mathrm{~ms}$ delay (4), and with inhibitory synapses to the retractors. This increases the joint torque sent to the colliding whiskers, in order to impede their rebound and to keep them at contact with the touched object.

In the $\mathrm{TN}$, we have included an additional population made of 20 neurons for each whisker, that has to encode the phase of the whisking period at which the contact occurs. TG has been implemented as an array of coincidence detectors (phase decoder), one for each TG whisking neuron, gating them in a logical AND with TG pressure neurons. The result is a labelled-line encoding of contact phase. The phase decoder is implemented by a transfer function that takes input from afferents in TG (pressure and whisking cells) and projects to the TN phase population in the trigeminal nuclei (Figure 1C). For each TG whisking cell, the same spike rate is propagated downward only if pressure cells are firing. The phase information is needed for the precise localization, with respect to the mouse head, of the object touched by the whiskers.

Table 1 summarizes the connectivity between the different populations of the whisker system.

Testing protocol. The peripheral components of the whisker system described above has been tested inside the Neurorobotics Platform in free whisking conditions. The mouse moved its whiskers in an empty environment or touching an object and the spiking activity of FN, TG and TN has been recorded in order to verify the proper functioning of the developed system.

Experimental whisking-based object localization task. To provide a meaningful example of how the developed mouse whisker system can be used to build in-silico neurorobotic experiments, we reproduced an experimental study which investigated the involvement of the cerebellum in a whiskingbased object localization task in head-fixed mice (33). Rahmati and colleagues tested two populations of mice, one wildtype (Control) and one knock-out (L7-PP2B), suffering from genetically impaired cerebellar plasticity. Water-deprived mice had to learn to locate a vertical bar in their whisker field and lick a water droplet (GO trial) within a time response window or to refrain from licking (NOGO trial) according to the bar position.

During the first sessions, both mouse populations started with high hit rates and high false alarm rates. Control mice showed faster learning capabilities, reducing their licking response to NOGO trials after the first 4 training sessions. Conversely, knock-out mice reduced their licking in a random fashion staying close to the guess rate (33). Therefore, they concluded that cerebellar plasticity has a crucial role in this sophisticated cognitive task requiring strict temporal processing. 
bioRxiv preprint doi: https://doi.org/10.1101/2021.10.26.465919; this version posted October 28, 2021. The copyright holder for this preprint (which was not certified by peer review) is the author/funder, who has granted bioRxiv a license to display the preprint in perpetuity. It is made available under aCC-BY-NC 4.0 International license.

Table 1. Connectivity of the SNN whisker system model

\begin{tabular}{l|c|c|c|c} 
Synaptic Connection & Type & Number & Convergence & Divergence \\
\hline \hline CPG-FN protractors & Excitatory & 80 & 1 & 80 \\
CPG-FN retractors & Excitatory & 40 & 1 & 40 \\
TG contact-TN contact & Excitatory & 80 & 20 & 1 \\
TN contact-FN protractors & Excitatory & 80 & 1 & 20 \\
TN contact-FN retractors & Inhibitory & 80 & 1 & 20 \\
\hline \hline
\end{tabular}

Cerebellar SNN model. To investigate in-silico the role of cerebellar plasticity during the task, we integrated a well established cerebellar-inspired SNN model to the whisker system described above. Recently, a detailed spiking neural network model of the cerebellar microcircuit proved able to reproduce multiple cerebellar-driven tasks $(26,34-37)$. Here, we used the model to drive learning in the in-silico whiskingbased object localization task.

The SNN cerebellar microcircuit (Figure 2) was populated with leaky Integrate\&Fire neurons, distinguishing between different neural groups. Mossy Fibers (MFs), the input to the cerebellar module, encode the state of the body-environment system: the whisker current position and the localization of an eventual object, e.g., the cue signalling a GO trial. Therefore, MFs receive excitatory connections from TG pressure cells and TN phase cells. Granular Cells (GrCs) represent in a sparse way the input from the MFs. Inferior Olive neurons (IOs), the other input to the cerebellar module, encode the reward, which is provided when a response is correctly generated (i.e., in a GO trial). In fact, this neural population responds to attention or surprise signals. Purkinje Cells (PCs) integrate the sparse information coming from the $\mathrm{GrCs}$ through the Parallel Fibers (PFs), while Deep Cerebellar Nuclei (DCN), the only output of the cerebellar module, generate the response (i.e., the equivalent of licking). The firing rate of DCN is monitored and a response is detected when the firing rate exceeds a pre-defined threshold (i.e., $80 \mathrm{~Hz}$ ). The network structure and connectivity are reported in Figure 2 and Table 2.

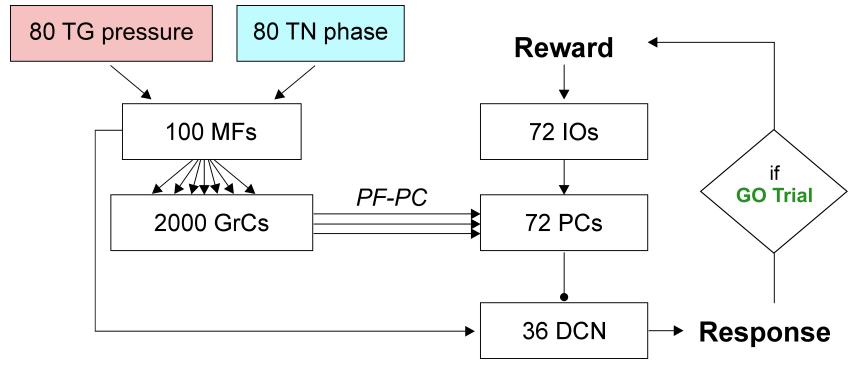

Fig. 2. SNN implementation of the cerebellum. Whisking sensory signals are conveyed to the cerebellar MFs from TG pressure and TN phase neurons, while the reward signal during correct GO trials reach the 10 neurons; the cerebellum controls the output motor response (head movement) according to DCN activity (i.e., generation of a response, head raise, when the firing rate exceeds a set threshold). Arrows and circles represent excitatory and inhibitory connections, respectively.

The cerebellar SNN model included one plasticity site, at the cortical level, between PFs and PCs, based on a wellknown kind of STDP (38-40). Synaptic weights between
PF-PC plasticity are modulated by IO activity (IOs-PCs connections in Table 2 are indicated as "teaching"), depending on the difference between the pre- and post-synaptic firing times $(36,41,42)$. Long-Term Potentiation (LTP) and LongTerm Depression (LTD) are the two possible changes that each synaptic connection can undergo. Synaptic weights increase (LTP) whenever a PC only receives an input from a PF, while they decrease (LTD) when associated with IO inputs (43-47). The learning rule can be formalized as in (2).

$$
\begin{gathered}
\Delta W_{P F_{i} \rightarrow P C_{j}}(t)= \\
\begin{cases}L_{I T D}^{t_{\text {IOspike }}} \int_{-\infty} K(t-x) \delta_{P F_{i}}(t-x) \mathrm{d} x & \text { if } P C_{j} \text { active } \\
& t=t_{\text {IOspike }_{j}} \\
L T P & \text { if } P C_{j} \text { active } \\
& t \neq t_{\text {IOspike }} \\
0 & \text { otherwise }\end{cases}
\end{gathered}
$$

where:

$$
\delta_{P F_{i}}(s)= \begin{cases}1 & \text { if } \mathrm{PF}_{i} \text { is active at time } s \\ 0 & \text { otherwise }\end{cases}
$$

and the kernel function is:

$$
K(z)=\mathrm{e}^{-\left(z-t_{0}\right)}\left(\sin \left(2 \pi\left(z-t_{0}\right)\right)\right)^{20}
$$

where $t_{I O \text { spike }_{j}}$ is the time when $I O_{j}$ emits a spike; $K(z)$ is the integral kernel function, which has its peak at $t_{0}(100$ ms) before $t_{I O s p i k e}$. The plastic learning rule is characterized by two constants, $L T P$ and $L T D$, which regulate the amount of synaptic change. These constants cannot be directly computed from physiological data, but they have been set to values found in related modelling studies $(L T P=0.01$, $L T D=-0.03)(37)$.

Neurorobotic implementation of the whisking-based object localization task. In the NRP, a licking-like movement, has been set for the virtual mouse: it has to rise its head and touch a shelf positioned just above. During GO trials, a vertical bar is displaced in the left whisker field and if the mouse rises its head, a reward signal is triggered. During NOGO trials the bar is on the right and if the mouse rises its head it does not receive any reward (Figure $3 \mathrm{~A}$ ).

The experiment is composed of short trials of 2 seconds, divided in GO and NOGO trials. The vertical bar is displayed 
Table 2. Connectivity of the SNN cerebellar model

\begin{tabular}{l|c|c|c|c} 
Synaptic Connection & Type & Number & Convergence & Divergence \\
\hline \hline TG pressure-MFs & Excitatory & 80 & 1 & 1 \\
TN phase-MFs & Excitatory & 80 & 1 & 1 \\
MFs-GrCs & Excitatory & 8000 & 4 & 80 \\
PFs-PCs & Excitatory & 115200 & 1600 & 58 \\
IOs-PCs & Teaching & 72 & 1 & 1 \\
MFs-DCN & Excitatory & 3600 & 100 & 36 \\
PCs-DCN & Inhibitory & 72 & 2 & 1 \\
\hline \hline
\end{tabular}

in the mouse whisker field for 1 second, while the response window continue till the end of the trial.

Trials were grouped in sessions composed of 10 trials, 5 GO and 5 NOGO, performed in a randomized sequence. The neurorobotic experiment included 27 sessions, following the experimental protocol. In order to evaluate the learning of the controller, for each session we recorded the percentage of correct responses in GO trials ("hit rate"), and the percentage of responses in NOGO trials ("false alarms").

Experimental and in-silico cerebellar impairment. PCspecific PP2B knock-out (L7-PP2B) mice show deficits in motor learning, consolidation, and procedural learning $(48,49)$ while they behave normally in standard non-motor tasks (50). In their experiment, Rahmati and colleagues tested how an impairment of the PF-PC LTP influenced the performance in the whisking-based object localization task. They demonstrated that learning in L7-PP2B mice was severely impaired, indicating that this task can depend, at least to some extent, on cerebellar plasticity.

We recreated in-silico the impaired cerebellum dramatically reducing the constant $L T P$ (see (2)) to $10 \%\left(L T P_{L 7-P P 2 B}\right.$ $=0.001$ ). We repeated each experiment (i.e., 27 sessions, 10 trials each, therefore 270 trials) of the localization protocol 10 times, using the impaired cerebellar model, then we compared the curves of hit rate and false alarms between L7PP2B and control mice.

Hardware and software. For the simulations, we have used a local installation of the NRP version 3.1, exploiting Python 3.8 (RRID:SCR_008394), Gazebo 11 (51), and ROS Noetic (52).

The simulation of the controller has been done with NEST, a software simulator for spiking neural networks (53-55). We used NEST 2.18 (56) (RRID:SCR_002963), interfaced through PyNN 0.9.5 (57) (RRID:SCR_002963).

All the simulations have been carried out on a Desktop PC provided with Intel Core i7-2600 CPU @ $3.40 \mathrm{GHz}$ and 16 GB of RAM, running 64 bit Ubuntu 20.04.2 LTS.

\section{Results}

We successfully developed a SNN model of the sensorimotor peripheral whisker system, modelling trigeminal ganglion, trigeminal nuclei, facial nuclei, and central pattern generator neuronal populations. This peripheral SNN was embedded in a virtual mouse robot, and it was properly connected to an adaptive cerebellar SNN. The whole system was able to drive active whisking with learning capability, matching neural correlates of behaviour experimentally recorded in mice.

Motor pathway. The four whiskers are controlled by the motoneurons present in the FN. They are working under the control of a single CPG neuron, firing at $4 \mathrm{~Hz}$, which rhythmically excites protractors and retractors neurons. Motoneurons spikes are then transformed into torques applied independently at each whisker. As shown in Figure 3B, during a free whisking period, the spiking pattern of the four groups of neurons is very similar, with a precise temporal alternation between protractors, causing the whisker to move forward, and retractors, pulling the whiskers back to the initial position. Whiskers' movements are slightly shifted with respect to the spikes due to the delays introduced by the conversion between spikes and torques and by the mechanical inertia of the whiskers. The mean firing rate of protractors and retractors neurons are $3.80 \mathrm{~Hz}$ and $4.00 \mathrm{~Hz}$, respectively, with a peak firing rate of $48.75 \mathrm{~Hz}$ and $55.00 \mathrm{~Hz}$, respectively. Figure $3 \mathrm{C}$ shows how the whisker trajectory changes during GO and NOGO trials. Namely, in GO trials a bar is placed for 1 second in the left whisker field, therefore whiskers L0 and L1 hit it and their range of motion is therefore reduced to $\sim 15$ degrees. The same behaviour can be observed during NOGO trials for whiskers R0 and R1.

Sensory pathway. Neurons in the TG and TN compose the sensory pathway and the first stage of elaboration is done by TG neurons. Each group of TG shows specific activity patterns depending on its function (Figure 4A). TG whisking neurons follow the angular profile of each of the four whiskers; it is possible to notice the differences between GO and NOGO trials, where left and right whiskers change their spatial profile when hitting the bar in the first second of each trial. Their mean firing rate is $3.65( \pm 1.93) \mathrm{Hz}$. TG contact neurons fire at $\sim 5 \mathrm{~Hz}$ when the whisker hits the bar, while TG detach neurons when the whisker is no longer in contact with the bar, because the bar has been removed or because the whisker has been retracted. TG pressure neurons are active for the whole duration of the contact between the whisker and the bar $(27.00 \pm 17.69 \mathrm{~Hz})$. In the SNN model, we have included an additional population, TG high threshold neurons, which are activated when the contact happens close to the nose of the robot (less than $2 \mathrm{~cm}$ ). However, in our protocol 

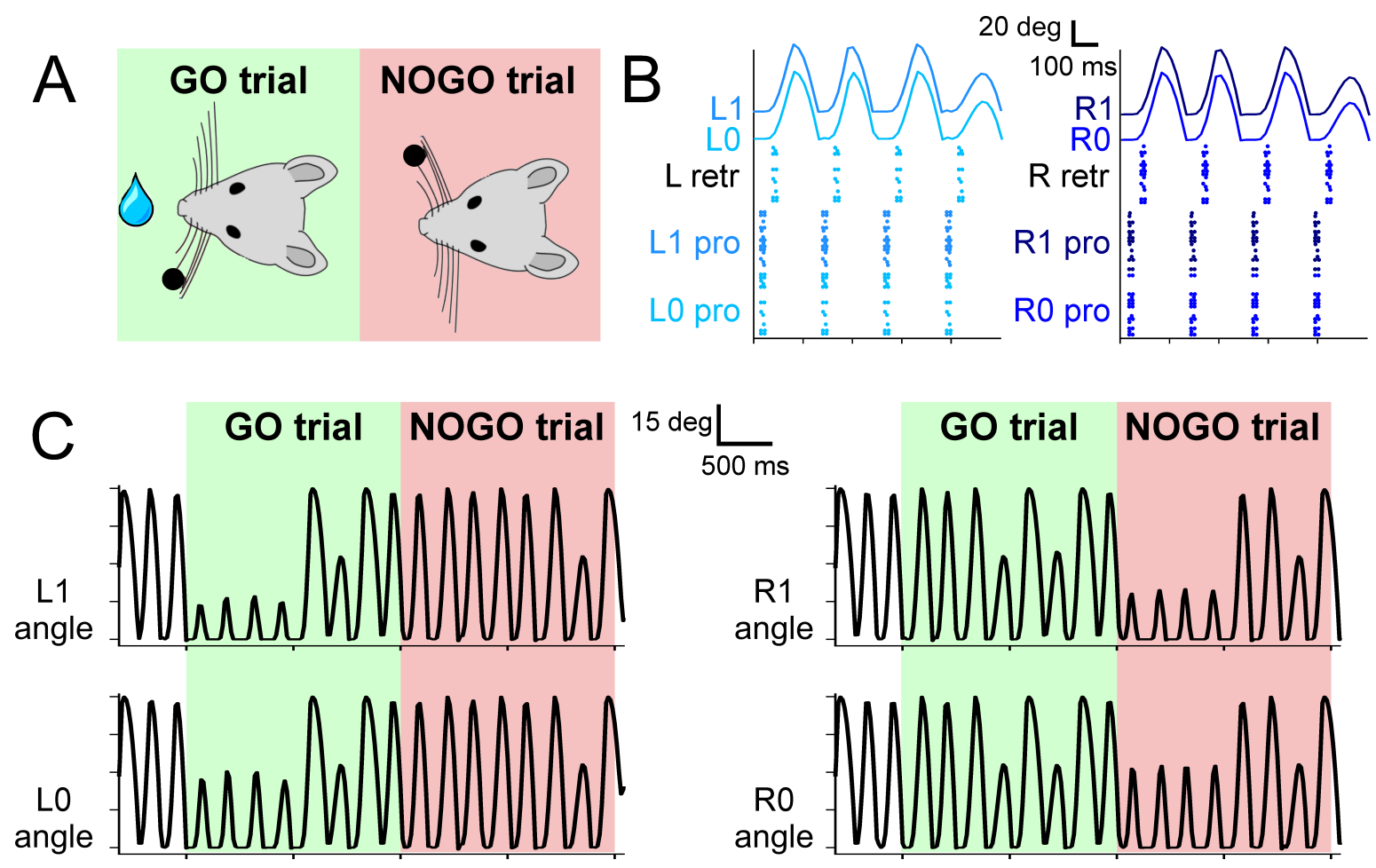

Fig. 3. A) experimental protocol, during GO trials, a sensory cue (a small bar, depicted as a black dot) is placed in the left whisker field of the mouse. Correct responses lead to a reward (water drop). During NOGO trials, the sensory cue is placed in the right whisker field and a response does not result in any reward. B) Spiking activity of motor neurons for protraction and retraction during one trial. 20 protractors neurons for each whisker and 20 retractor neurons for each side ( $L$ and $R$ ) fire under the control of the $\mathrm{CPG}$ neuron at $4 \mathrm{~Hz}$. The resulting displacement of each whisker is depicted in the upper part of the panel. C) Angular displacement of the four whiskers during one GO and one NOGO trial. During the GO trial, in the first second, left whiskers hit the sensory cue bar, placed in the left whisker field. On the other hand, during the NOGO trial, the right whiskers hit the sensory cue bar placed in the right whisker field.

the bar is placed at a higher distance, therefore those neurons were never activated.

Figures $4 \mathrm{~B}$ and $4 \mathrm{C}$ provide a direct comparison between the firing rates of the different $\mathrm{TG}$ populations during one whisker movement. Figure 4B has been adapted from (29), while in Figure $3 \mathrm{C}$ the firing rates of the neurons in a specific trial (the magnified inset from Figure 4A) have been computed with bins of $10 \mathrm{~ms}$. It is possible to appreciate that insilico TG neurons show a behaviour comparable to the one of biological neurons, especially for the timing of their response with respect to the events of protraction, contact, detach, and retraction.

Learning performance. We have shown that the SNN representing the sensorimotor whisker system is able to encode in a biologically realistic way the sensory and motor signals exchanged with a robotic plant. To demonstrate how this system can be used to recreate a complex behavioural test, we connected the whisking sensory system to an adaptive SNN and we challenged the integrated system in the object localisation experiment proposed by Rahmati and colleagues (33). The aim of the mouse is to lick during the GO trials and to refrain from licking during the NOGO trials, distinguishing between the two conditions according to the position of a bar placed into their whisker field. Figure 5B reports the percentages of correct licks in GO trials and the number of incorrect licks in NOGO trials. The reference behavioural data recorded in animals are reported in Figure 5A.
Considering control animals, it is possible to see that mice lick continuously in the first sessions (i.e., hit rate and false alarms are both close to $100 \%$ ), without distinguishing GO and NOGO trials. While the experiment proceeds, the animals learn to refrain from licking during the NOGO trials. In fact, the percentage of false alarms decreases toward $0 \%$. At the same time, the percentage of correct licks remains close to $100 \%$.

Control and L7-PP2B mice differed in their learning skills. Both started the training with high hit and false alarm rates. As a result, they performed close to the guess rate. During the subsequent sessions, control mice consistently increased accuracy, specifically reducing their response to NOGO trials. In contrast, L7-PP2B mice continue for more sessions to not discriminate between $\mathrm{GO}$ and NOGO trials. In the later sessions, also L7-PP2B diminished their licks in the NOGO trials, but their learning trajectories remained noisier than those of controls, taking a longer time to reach high performance levels. This observation suggested that a functional LTP mechanism was important to obtain a superior ability to rapidly discriminate between GO and NOGO cues, and then respond accordingly.

The performances and the learning trajectories of control and L7-PP2B in-silico models (Figure 5B) are similar to their biological counterparts. While both models maintain a perfect recognition of GO trials, the control model learned to refrain from licking in NOGO trial faster and stably. The variability present between the 10 different tests is due to the different 

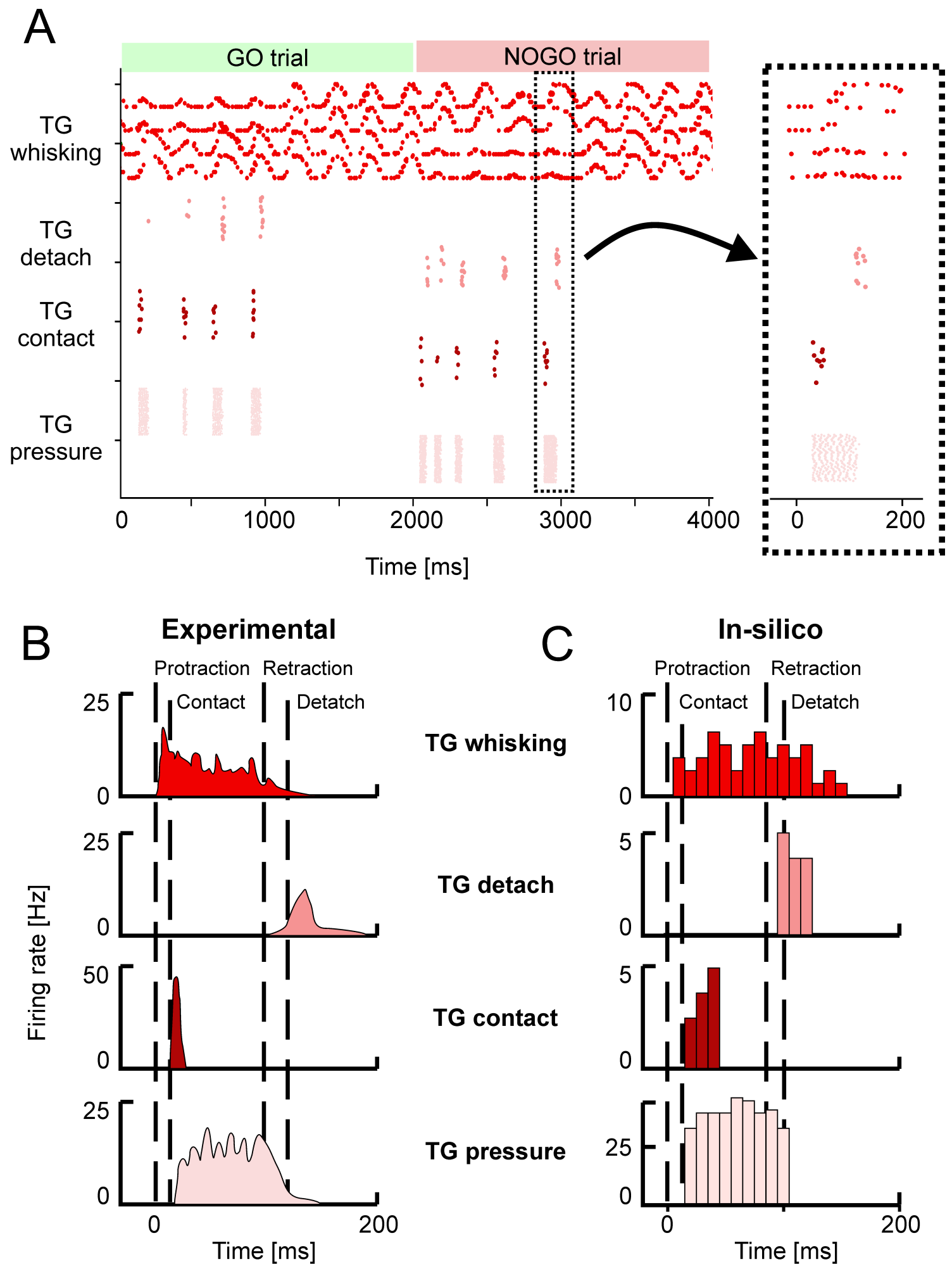

Fig. 4. A) Spiking activity of the Trigeminal Ganglion (TG) neurons during GO and NOGO trials. Each row represents the activity of one neuron, different shades of red are used to plot the activity of the four groups of TG neurons. The inset shows a magnified portion of the full scatterplot, focusing on a single protraction-retraction movement of the whiskers. (B) Firing rates of TG populations measured experimentally during a single protraction-retraction movement, as reported in (29). Vertical dashed lines represent the four main events: start of the protraction, contact of the whisker against an object, start of the retraction, detach of the whisker from the object. (C) Firing rates recorded from the simulation of the SNN model of TG populations. The length of each bin is $10 \mathrm{~ms}$. Colors are the same as panels A and B.

sequences of GO and NOGO trials, which were randomly extracted for each session.

Looking at the spiking activity of the cerebellar network, and in particular at the DCN population (Figure 5C), which drives the response of the mouse, it is possible to appreciate the different evolution in the spiking patterns. Control and L7-PP2B simulations have a similar activity in the DCN during the 10 trials of the first session, the neurons fire regardless of the in- 
put arriving from the whisker system (right or left contacts) and therefore DCN generate a response during both GO and NOGO trials. After 10 sessions of training, the Control simulation shows intense DCN activity during GO trial and weak or null activity during NOGO trials, proving that the cerebellar network has learned the association between left/right stimulation with the presence/absence of the reward. This behaviour is impaired for the L7-PP2B simulation, in fact, there are still several DCN that are firing during both GO and NOGO trials, then causing a high False alarm rate.

Each session took about 140 minutes for a simulated time of 540 seconds (i.e., 270 trials of 2 seconds each), with a scaleup with respect to the real-time equal to $\sim 15$ times. The maximum RAM consumption was equal to $\sim 15 \mathrm{~GB}$.

\section{Discussion and conclusions}

We developed a spiking neural model of the mouse whisker system, covering both sensory and motor pathways, and their interconnections. The implemented system takes into account the different roles that groups of cells have at the different stages of the sensorimotor processing, providing coding for complex information such as the object localization performed during the active whisking. This system, properly connected to an adaptive cerebellar-inspired spiking network, reproduced complex in-vivo experiments, by using the neurorobotics platform.

The peripheral whisker system showed appropriate discharge patterns as in in-vivo experimental recordings during whisking, in precise time-windows of exploration and object interaction and depending on which side the stimulus was presented within the whisker field.

The peripheral system, when wired to a cerebellar SNN with plasticity and tested in an object-localization task, was able to reduce the number of useless responses along a sequence of trials (triggered by the NOGO trials) which did not correspond to any reward. This learning curve was slowed down when the plasticity parameter (LTP rate) of the cerebellar SNN was strongly reduced, as in knock-out mice recorded experimentally.

The integrated circuit, entirely made of spiking neurons, proved the good integration of different ways of neural coding. In fact, while the main parameter correlating response patterns to behavior was the average firing frequency of the DCN population, other elements of the whisker system used a variety of encoding strategies. For instance, the time-coded activity of TG contact and TG detach cells or the TG whisking cells encoding the current whisker position by means of their Gaussian-shaped sensitivity.

The model here proposed can be used as a reference for future advanced neurorobots and for neuroscience in-silico experiments, to investigate the role of cerebro-cerebellar loops and cerebellar physiology in whisking protocols.

Limitations and future challenges. Considering the robotic aspects, a limitation of the physical simulator (Gazebo) regards the properties of the materials that can be used. Rodents rely on whiskers bending to recognise the shape of objects and on their resonance frequencies to detect textures $(58,59)$, but the current state of the simulators used by the NRP supports only rigid bodies. Using only rigid whiskers makes object recognition tasks more difficult, unless maybe using large arrays of finely spaced whiskers. Therefore, this work focused on extracting only spatial information, which can be easily performed with just rigid whiskers, and not more sophisticated features of the touched object.

Much of the work on the whisker system consisted in the encoding of information in TG primary afferents, ignoring all of the internal brain structures involved in the whisker system, in particular the somatotopic mapping emerging in the $\mathrm{TN}$ and propagated in the thalamo-cortical system. Loops between thalamus and cortex have been cited as possible location for mechanisms decoding phase information, with the use of neuronal phase-locking loops. A possible future development can be exploring other loops in the brainstem outside the TN, such as the ones involving the superior colliculus and their interactions with attention and foveation (60).

The work on the cerebellar control mechanisms was mainly limited by the long simulation times of the NRP, which influenced the choice of network and learning parameters. Given the limited number of mossy fibers and granule cells (100 and 2000, respectively), the cerebellar network showed a reduced generalization capability. The discrimination task, in this case, was between two very different conditions (object hit with the left or with the right whiskers). Mice have a higher resolution, since they can recognize objects slightly moved or even objects with different textures. With larger populations, training could make different sub-populations respond to different inputs, encoding for more complex features of the sensed environment. Future work can explore this hypothesis making rigorous analyses on cell responses and optimizing the network size to the variety of input patterns.

We have chosen I\&F neuron models as building blocks of the SNN network. However, nowadays, there are much more complex models, taking into account many mechanisms related to membrane potential and ionic currents (biophysical models (61)) or more advanced I\&F neuron models $(62,63)$. Even though these models are more accurate representations of the biological elements, their complexity would require too much computational power to simulate a network made of thousands of neurons, which would eventually prevent from embedding these models as controllers in neurorobots. Therefore, the model we are proposing is a trade-off between computational efficiency and biological realism.

NEST-based simulations offer a great possibility to develop and test biologically inspired models, but require high-performance computing for large-scale models (64) and therefore does not allow performances sufficient to control robots in real world situations. This could allow testing the robustness of the bioinspired controller against common environmental noise, increasing the similarity with experimental results (e.g., the imperfect $(<100 \%)$ hit rates accuracies achieved by experimental animals). An already available so- 

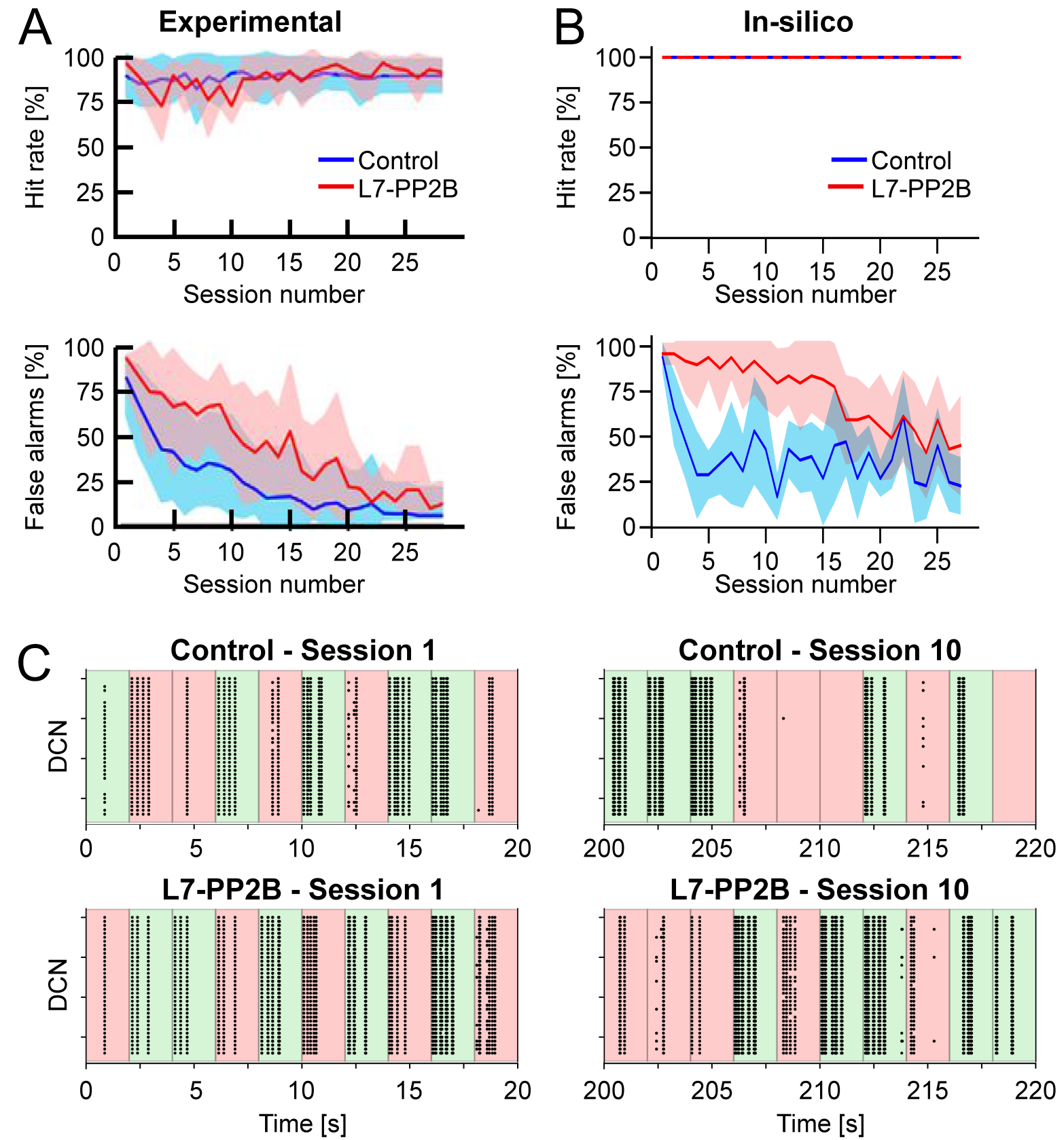

Fig. 5. A) Learning curves recorded in the experiment performed by Rahmati and colleagues (33). The upper row shows the Hit rate (i.e., the percentage of correct responses in GO trials) along sessions, where each session is composed of 10 trials. The lower row shows the False alarms (i.e., the percentage of incorrect responses in NOGO trials) along sessions. The blue and red curves show the mean values for control animals and knock-out (L7-PP2B) mice. Shaded areas show the standard deviation. B) Learning curves recorded from the in-silico experiments (10 control and 10 knock-out models). Colors are the same as in panel A. C) Spiking activity of the DCN neurons during GO (green) and NOGO (red) trials, in the first session (left column) and after 10 sessions of training (right column). The first row reports the activity of one Control simulation, while the second row reports one knock-out simulation. Each dot is a spike of one of the $36 \mathrm{DCN}$ in the cerebellar network. The order of GO and NOGO trials is randomized for each session and simulation, but all sessions have 5 GO and 50 NOGO trials.

lution to achieve real-time performances can be to rely on spiking neural networks running on neuromorphic hardware. Very recently, a cerebellar-inspired model made of 97 thousand neurons and 4.2 million synapses has been implemented on the neuromorphic platform SpiNNaker (65). This type of solution could be applicable if the plasticity rule used at PFPC synapses, supervised by IO activity, will be implemented on this or other neuromorphic systems. On the other hand, the SNN whisker system here presented can be simulated on SpiNNaker chips, since it has been developed using PyNN, which supports both NEST and SpiNNaker as simulators.

Conclusions. Neuroscientists have not fully uncovered the neural mechanisms for mouse whisking, but it is clear that it involves a complex architecture composed of multiple sensorimotor loops. In this work, we developed and tested a spiking computational model of the peripheral whisker system, reproducing the neural dynamics observed in its different components and embedded in a virtual mouse neurorobot controlled by a cerebellar SNN. 
bioRxiv preprint doi: https://doi.org/10.1101/2021.10.26.465919; this version posted October 28, 2021. The copyright holder for this preprint (which was not certified by peer review) is the author/funder, who has granted bioRxiv a license to display the preprint in perpetuity. It is made available under aCC-BY-NC 4.0 International license.

The virtual mouse enriched with this peripheral whisker system may be connected to more realistic multi-area brain models, to shed light on how these regions together may control the precise timing of whisker movements and coordinate whisker perception.

In the future, refined versions of the model could exhibit more advanced features, such as the recognition of surface textures, identification of movements of the touched object, or other complex touch-guided behaviours. From a technological perspective, neuromorphic implementations can be employed to speed up the computation until reaching realtime performances, granting the possibility to embed the whisker system in physical robots.

\section{Conflict of Interest Statement}

The Authors declare that the research was conducted in the absence of any commercial or financial relationships that could be construed as a potential conflict of interest.

\section{Author Contributions}

AA, AG, CC designed the model and the computational framework. AA, AG, EN carried out the implementation. $\mathrm{AA}$ and EN wrote the manuscript with input from all authors. AA, EDA, CC, AP conceived the study and were in charge of overall direction and planning. All authors discussed the results and commented on the manuscript.

\section{Funding}

This research received funding from the European Union's Horizon 2020 Framework Program for Research and Innovation under the Specific Grant Agreement No. 785907 (Human Brain Project SGA2) and Specific Grant Agreement No. 945539 (Human Brain Project SGA3).

\section{Data Availability Statement}

The computational models used for the simulation are publicly available at https://github.com/albertoantonietti/paper_whisking. Data generated from the simulations and Python codes to generate all the figures presented in this work are available in the same GitHub repository.

1. Mehdi Adibi. Whisker-Mediated Touch System in Rodents: From Neuron to Behavior. Frontiers in Systems Neuroscience, 13(August):1-24, 2019. ISSN 16625137. doi: 10.3389/fnsys.2019.00040.

2. Jeffrey D Moore, Martin Deschênes, Anastasia Kurnikova, and David Kleinfeld. Activation and measurement of free whisking in the lightly anesthetized rodent. Nature Protocols, 9 (8):1792-1802, jul 2014. doi: 10.1038/nprot.2014.119.

3. Quoc-Thang Nguyen and David Kleinfeld. Positive feedback in a brainstem tactile sensorimotor loop. Neuron, 45(3):447-457, feb 2005. doi: 10.1016/..neuron.2004.12.042.

4. Marie-Andrée Bellavance, Jun Takatoh, Jinghao Lu, Maxime Demers, David Kleinfeld, Fan Wang, and Martin Deschênes. Parallel inhibitory and excitatory trigemino-facial feedback circuitry for reflexive vibrissa movement. Neuron, 95(3):722-723, aug 2017. doi: 10.1016/j. neuron.2017.07.022.

5. Laurens W. J. Bosman, Arthur R. Houweling, Cullen B. Owens, Nouk Tanke, Olesya T. Shevchouk, Negah Rahmati, Wouter H. T. Teunissen, Chiheng Ju, Wei Gong, Sebastiaan K. E. Koekkoek, and Chris I. De Zeeuw. Anatomical pathways involved in generating and sensing rhythmic whisker movements. Frontiers in Integrative Neuroscience, 5, 2011. doi: 10.3389/fnint.2011.00053.

6. Lauren E. McElvain, Beth Friedman, Harvey J. Karten, Karel Svoboda, Fan Wang, Martin Deschênes, and David Kleinfeld. Circuits in the rodent brainstem that control whisking in concert with other orofacial motor actions. Neuroscience, 368:152-170, jan 2018. doi: 10.1016/j.neuroscience.2017.08.034.
7. Romain Brette. Philosophy of the spike: Rate-based vs. spike-based theories of the brain. Frontiers in Systems Neuroscience, 9, nov 2015. doi: 10.3389/fnsys.2015.00151.

8. Samanwoy Ghosh-Dastidar and Hojjat Adeli. A new supervised learning algorithm for multiple spiking neural networks with application in epilepsy and seizure detection. Neural Networks, 22(10):1419-1431, dec 2009. doi: 10.1016/j.neunet.2009.04.003.

9. Filip Ponulak and Andrzej Kasiński. Introduction to spiking neural networks: Information processing, learning and applications. Acta Neurobiologiae Experimentalis, 2011. ISSN 00651400 .

10. Amirhossein Tavanaei, Masoud Ghodrati, Saeed Reza Kheradpisheh, Timothée Masquelier, and Anthony Maida. Deep learning in spiking neural networks. Neural Networks, 111: 47-63, 2019.

11. Eugene M Izhikevich and Niraj S Desai. Relating stdp to bcm. Neural computation, 15(7): 1511-1523, 2003.

12. Eugene M Izhikevich. Solving the distal reward problem through linkage of stdp and dopamine signaling. Cerebral cortex, 17(10):2443-2452, 2007.

13. Henry Markram, Wulfram Gerstner, and Per Jesper Sjöström. A history of spike-timingdependent plasticity. Frontiers in synaptic neuroscience, 3:4, 2011.

14. Henry Markram, Wulfram Gerstner, and Per Jesper Sjöström. Spike-timing-dependent plasticity: a comprehensive overview. Frontiers in synaptic neuroscience, 4:2, 2012.

15. Vincent Delattre, Daniel Keller, Matthew Perich, Henry Markram, and Eilif Benjamin Muller. Network-timing-dependent plasticity. Frontiers in cellular neuroscience, 9:220, 2015.

16. Alois Knoll. Plenary talk 1: Bionic systems: Basic research and recent results in neurorobotics in the human brain project. In 2017 IEEE International Conference on Cyborg and Bionic Systems (CBS). IEEE, oct 2017. doi: 10.1109/cbs.2017.8266059.

17. Susu Chen, George J. Augustine, and Paul Chadderton. Serial processing of kinematic signals by cerebellar circuitry during voluntary whisking. Nature Communications, 8(1), aug 2017. doi: 10.1038/s41467-017-00312-1.

18. T. Pipe and M. J. Pearson. Whiskered robots. Scholarpedia, 10(3):6641, 2015. doi: 10. 4249/scholarpedia.6641. revision \#150527.

19. Martin J. Pearson, Anthony G. Pipe, Chris Melhuish, Ben Mitchinson, and Tony J. Prescott. Whiskerbot: A robotic active touch system modeled on the rat whisker sensory system. Adaptive Behavior, 15(3):223-240, sep 2007. doi: 10.1177/1059712307082089.

20. Martin J. Pearson, Ben Mitchinson, Jason Welsby, Tony Pipe, and Tony J. Prescott. SCRATCHbot: Active tactile sensing in a whiskered mobile robot. In From Animals to Animats 11, pages 93-103. Springer Berlin Heidelberg, 2010. doi: 10.1007/ 978-3-642-15193-4_9.

21. M. J. Pearson, B. Mitchinson, J. C. Sullivan, A. G. Pipe, and T. J. Prescott. Biomimetic vibrissal sensing for robots. Philosophical Transactions of the Royal Society B: Biological Sciences, 366(1581):3085-3096, oct 2011. doi: 10.1098/rstb.2011.0164.

22. J. Charles Sullivan, Ben Mitchinson, Martin J. Pearson, Mat Evans, Nathan F. Lepora, Charles W. Fox, Chris Melhuish, and Tony J. Prescott. Tactile discrimination using active whisker sensors. IEEE Sensors Journal, 12(2):350-362, feb 2012. doi: 10.1109/jsen.2011. 2148114.

23. Egidio Falotico and et al. Connecting artificial brains to robots in a comprehensive simulation framework: the neurorobotics platform. Frontiers in neurorobotics, 11:2, 2017. doi: 10.3389/ fnbot.2017.00002.

24. Lorenzo Vannucci, Egidio Falotico, Silvia Tolu, Vito Cacucciolo, Paolo Dario, Henrik Hautop Lund, and Cecilia Laschi. A comprehensive gaze stabilization controller based on cerebellar internal models. Bioinspiration \& Biomimetics, 2017.

25. Alban Bornet, Jacques Kaiser, Alexander Kroner, Egidio Falotico, Alessandro Ambrosano, Kepa Cantero, Michael H Herzog, and Gregory Francis. Running Large-Scale Simulations on the Neurorobotics Platform to Understand Vision - The Case of Visual Crowding. Frontiers in neurorobotics, 13, 2019. ISSN 1662-5218. doi: 10.3389/fnbot.2019.00033.

26. Carlos Corchado, Alberto Antonietti, Marie Claire Capolei, Claudia Casellato, and Silvia Tolu. Integration of paired spiking cerebellar models for voluntary movement adaptation in a closed-loop neuro-robotic experiment. A simulation study. In 2019 IEEE International Conference on Cyborg and Bionic Systems (CBS), pages 110-115. IEEE, sep 2019. ISBN 978-1-7281-5073-4. doi: 10.1109/CBS46900.2019.9114412.

27. R.N. Van Horn. Vibrissae structure in the rhesus monkey. Folia Primatologica, 13(4):241285, 1970. doi: 10.1159/000155325.

28. Michael Brecht, Bruno Preilowski, and Michael M. Merzenich. Functional architecture of the mystacial vibrissae. Behavioural Brain Research, 84(1-2):81-97, mar 1997. doi: 10.1016/ s0166-4328(97)83328-1.

29. Ehud Ahissar and Per M. Knutsen. Vibrissal Location Coding, pages 725-735. Atlantis Press, Paris, 2016. ISBN 978-94-6239-133-8. doi: 10.2991/978-94-6239-133-8_53.

30. Steven C. Leiser and Karen A. Moxon. Responses of trigeminal ganglion neurons during natural whisking behaviors in the awake rat. Neuron, 53(1):117-133, jan 2007. doi: 10. 1016/j.neuron.2006.10.036.

31. Marcin Szwed, Knarik Bagdasarian, and Ehud Ahissar. Encoding of vibrissal active touch. Neuron, 40(3):621-630, oct 2003. doi: 10.1016/s0896-6273(03)00671-8.

32. Cornelius Schwarz and Shubhodeep Chakrabarti. Whisking control by motor cortex. Scholarpedia, 10(3):7466, 2015. doi: 10.4249/scholarpedia.7466.

33. N. Rahmati, C. B. Owens, L. W. J. Bosman, J. K. Spanke, S. Lindeman, W. Gong, J.-W. Potters, V. Romano, K. Voges, L. Moscato, S. K. E. Koekkoek, M. Negrello, and C. I. De Zeeuw. Cerebellar potentiation and learning a whisker-based object localization task with a time response window. Journal of Neuroscience, 34(5):1949-1962, jan 2014. doi: 10.1523/ jneurosci.2966-13.2014.

34. Claudia Casellato, Alberto Antonietti, Jesús Garrido, Richard R Carrillo, Niceto R Luque, Eduardo Ros, Alessandra Pedrocchi, and Egidio D'Angelo. Adaptive robotic control driven by a versatile spiking cerebellar network. PloS one, 9:e112265, 11 2014. doi: 10.1371/ journal.pone. 0112265 .

35. Alberto Antonietti, Claudia Casellato, Jesus A. Garrido, Niceto R. Luque, Francisco Naveros, Eduardo Ros, Egidio DAngelo, and Alessandra Pedrocchi. Spiking neural network with distributed plasticity reproduces cerebellar learning in eye blink conditioning paradigms. IEEE Transactions on Biomedical Engineering, 63(1):210-219, jan 2016. doi: 10.1109/tbme.2015.2485301. 
bioRxiv preprint doi: https://doi.org/10.1101/2021.10.26.465919; this version posted October 28, 2021. The copyright holder for this preprint (which was not certified by peer review) is the author/funder, who has granted bioRxiv a license to display the preprint in perpetuity. It is made available under aCC-BY-NC 4.0 International license.

36. Alice Geminiani, Alessandra Pedrocchi, Egidio D'Angelo, and Claudia Casellato. Spiking cerebellar model with damaged cortical Neural population reproduces human ataxic behaviors in perturbed upper limb reaching. In 2017 8th International IEEE/EMBS Conference on Neural Engineering (NER), pages 489-492. IEEE, 2017. doi: 10.1109/NER.2017.8008396.

37. Alberto Antonietti, Dario Martina, Claudia Casellato, Egidio D'Angelo, and Alessandra Pedrocchi. Control of a Humanoid NAO Robot by an Adaptive Bioinspired Cerebellar Module in 3D Motion Tasks. Computational Intelligence and Neuroscience, 2019:1-15, jan 2019. ISSN 1687-5265. doi: 10.1155/2019/4862157.

38. Niceto R. Luque, Jesús A. Garrido, Francisco Naveros, Richard R. Carrillo, Egidio D’Angelo, and Eduardo Ros. Distributed cerebellar motor learning: A spike-timing-dependent plasticity model. Frontiers in Computational Neuroscience, 10, mar 2016. doi: 10.3389/fncom.2016. 00017.

39. Egidio D'Angelo, Alberto Antonietti, Stefano Casali, Claudia Casellato, Jesus A. Garrido, Niceto Rafael Luque, Lisa Mapelli, Stefano Masoli, Alessandra Pedrocchi, Francesca Prestori, Martina Francesca Rizza, and Eduardo Ros. Modeling the cerebellar microcircuit: New strategies for a long-standing issue. Frontiers in Cellular Neuroscience, 10, jul 2016. doi: 10.3389/fncel.2016.00176.

40. Niceto R Luque, Jesús A Garrido, Richard R Carrillo, Silvia Tolu, and Eduardo Ros. Adaptive cerebellar spiking model embedded in the control loop: context switching and robustness against noise. International journal of neural systems, 21(5):385-401, oct 2011. ISSN 0129-0657. doi: 10.1142/S0129065711002900.

41. Silvia Tolu, Mauricio Vanegas, Jesús A. Garrido, Niceto R. Lique, and Eduardo Ros. Adaptive and predictive control of a simulated robot arm. International Journal of Neural Systems, 23(03):1350010, April 2013. doi: 10.1142/s012906571350010x.

42. Ismael Baira Ojeda, Silvia Tolu, and Henrik H. Lund. A scalable neuro-inspired robot controller integrating a machine learning algorithm and a spiking cerebellar-like network. In Biomimetic and Biohybrid Systems, pages 375-386. Springer International Publishing, 2017. doi: 10.1007/978-3-319-63537-8_31.

43. Anders Rasmussen, Dan-Anders Jirenhed, Riccardo Zucca, Fredrik Johansson, Pär Svensson, and Germund Hesslow. Number of spikes in climbing fibers determines the direction of cerebellar learning. The Journal of Neuroscience, 33(33):13436-13440, aug 2013. ISSN 1529-2401. doi: 10.1523/JNEUROSCI.1527-13.2013.

44. Henrik Jörntell and Christian Hansel. Synaptic memories upside down: bidirectional plasticity at cerebellar parallel fiber-Purkinje cell synapses. Neuron, 52(2):227-38, oct 2006. ISSN 0896-6273. doi: 10.1016/j.neuron.2006.09.032.

45. Christian Hansel, David J Linden, and Egidio D'Angelo. Beyond parallel fiber LTD: the diversity of synaptic and non-synaptic plasticity in the cerebellum. Nature neuroscience, 4 (5):467-75, may 2001. ISSN 1097-6256. doi: 10.1038/87419.

46. Masao Ito, Kazuhiko Yamaguchi, Soichi Nagao, and Tadashi Yamazaki. Long-Term Depression as a Model of Cerebellar Plasticity, volume 210. Elsevier B.V., 1 edition, 2014. ISBN 9780444633569. doi: 10.1016/B978-0-444-63356-9.00001-7.

47. Eriola Hoxha, Filippo Tempia, Pellegrino Lippiello, and Maria Concetta Miniaci. Modulation, plasticity and pathophysiology of the parallel fiber-purkinje cell synapse. Frontiers in Synaptic Neuroscience, 8(November):1-16, 2016. ISSN 16633563. doi: 10.3389/fnsyn. 2016.00035.

48. Martijn Schonewille, A Belmeguenai, Sebastian K E Koekkoek, S H Houtman, Henk-Jan Boele, Boeke J van Beugen, Zhenyu Gao, Aleksandra Badura, G Ohtsuki, Wardell E Amerika, E Hosy, Freek E Hoebeek, Ype Elgersma, Christian Hansel, and Chris I De Zeeuw. Purkinje cell-specific knockout of the protein phosphatase PP2B impairs potentiation and cerebellar motor learning. Neuron, 67(4):618-28, aug 2010. ISSN 1097-4199. doi: 10.1016/j.neuron.2010.07.009.

49. Zhenyu Gao, Boeke J. van Beugen, and Chris I. De Zeeuw. Distributed synergistic plasticity and cerebellar learning. Nature Reviews Neuroscience, 13(9):619-635, sep 2012. ISSN 1471-0048. doi: 10.1038/nrn3312.

50. Elisa Galliano, Jan-Willem Potters, Ype Elgersma, William Wisden, Steven A Kushner, Chris I De Zeeuw, and Freek E Hoebeek. Synaptic transmission and plasticity at inputs to murine cerebellar Purkinje cells are largely dispensable for standard nonmotor tasks. The Journal of Neuroscience, 33(31):12599-618, jul 2013. ISSN 1529-2401. doi: 10.1523/JNEUROSCI.1642-13.2013.

51. C.E. Aguero, N. Koenig, I. Chen, H. Boyer, S. Peters, J. Hsu, B. Gerkey, S. Paepcke, J.L. Rivero, J. Manzo, E. Krotkov, and G. Pratt. Inside the virtual robotics challenge: Simulating real-time robotic disaster response. Automation Science and Engineering, IEEE Transactions on, 12(2):494-506, April 2015. ISSN 1545-5955. doi: 10.1109/TASE.2014.2368997.

52. Morgan Quigley, Brian Gerkey, Ken Conley, Josh Faust, Tully Foote, Jeremy Leibs, Eric Berger, Rob Wheeler, and Andrew Ng. Ros: an open-source robot operating system. In Proc. of the IEEE Intl. Conf. on Robotics and Automation (ICRA) Workshop on Open Source Robotics, Kobe, Japan, May 2009.

53. Marc-Oliver Gewaltig and Markus Diesmann. NEST (NEural simulation tool). Scholarpedia, 2(4):1430, 2007. doi: 10.4249/scholarpedia. 1430.

54. Jochen Martin Eppler, Moritz Helias, Eilif Muller, Markus Diesmann, and Marc-Oliver Gewaltig. PyNEST: A convenient interface to the NEST simulator. Frontiers in Neuroinformatics, 2(January):1-12, 2008. ISSN 16625196. doi: 10.3389/neuro.11.012.2008.

55. Hans Ekkehard Plesser, Markus Diesmann, Marc-Oliver Gewaltig, and Abigail Morrison. NEST: the Neural Simulation Tool. In Encyclopedia of Computational Neuroscience, pages 1849-1852. Springer New York, New York, NY, 2015. doi: 10.1007/978-1-4614-6675-8_258.

56. Jakob Jordan, Håkon Mørk, Stine Brekke Vennemo, Dennis Terhorst, Alexander Peyser, Tammo Ippen, Rajalekshmi Deepu, Jochen Martin Eppler, Alexander van Meegen, Susanne Kunkel, Ankur Sinha, Tanguy Fardet, Sandra Diaz, Abigail Morrison, Wolfram Schenck, David Dahmen, Jari Pronold, Jonas Stapmanns, Guido Trensch, Sebastian Spreizer, Jessica Mitchell, Steffen Graber, Johanna Senk, Charl Linssen, Jan Hahne, Alexey Serenko, Daniel Naoumenko, Eric Thomson, Itaru Kitayama, Sebastian Berns, and Hans Ekkehard Plesser. Nest 2.18.0, June 2019.

57. Andrew $\mathrm{P}$ Davison. PyNN: a common interface for neuronal network simulators. Frontiers in Neuroinformatics, 2, 2008. ISSN 16625196. doi: 10.3389/neuro.11.011.2008.

58. Lauren $\mathrm{M}$ Jones, Ernest Kwegyir-Afful, and Asaf Keller. Whisker primary afferents encode temporal frequency of moving gratings. Somatosensory \& motor research, 23:45-54, 07
2009. doi: 10.1080/08990220600702707.

59. Maria A. Neimark, Mark L. Andermann, John J. Hopfield, and Christopher I. Moore. Vibrissa resonance as a transduction mechanism for tactile encoding. Journal of Neuroscience, 23 (16):6499-6509, 2003. ISSN 0270-6474. doi: 10.1523/JNEUROSCI.23-16-06499.2003.

60. Miki Kaneshige, Ken-ichi Shibata, Jun Matsubayashi, Akira Mitani, and Takahiro Furuta. A descending circuit derived from the superior colliculus modulates vibrissal movements. Frontiers in Neural Circuits, 12:100, 2018. ISSN 1662-5110. doi: 10.3389/fncir.2018.00100.

61. M. L. Hines and N. T. Carnevale. Neuron: A tool for neuroscientists. The Neuroscientist, 7 (2):123-135, 2001. doi: 10.1177/107385840100700207. PMID: 11496923.

62. Eugene Izhikevich. Simple model of spiking neurons. IEEE Transactions on Neural Networks, 14(06), nov 2003. doi: 10.1109/TNN.2003.8204.

63. Alice Geminiani, Alessandra Pedrocchi, Egidio D'Angelo, and Claudia Casellato. Complex electroresponsive dynamics in olivocerebellar neurons represented with extendedgeneralized leaky integrate and fire models. Frontiers in Computational Neuroscience, 13 (35), 2019. doi: 10.3389/fncom.2019.00035.

64. Jakob Jordan, Tammo Ippen, Moritz Helias, Itaru Kitayama, Mitsuhisa Sato, Jun Igarashi, Markus Diesmann, and Susanne Kunkel. Extremely scalable spiking neuronal network simulation code: From laptops to exascale computers. Frontiers in Neuroinformatics, 12(02), 2018. doi: 10.3389/fninf.2018.00002.

65. Petruț Antoniu Bogdan, Beatrice Marcinno, Claudia Casellato, Stefano Casali, Andrew Rowley, Michael Hopkins, Francesco Leporati, Egidio D'Angelo, and Oliver Rhodes. Towards a bio-inspired real-time neuromorphic cerebellum. Frontiers in cellular neuroscience, March 2021. ISSN 1662-5102. doi: 10.3389/fncel.2021.622870. 